\title{
用于葡萄糖敏感药物释放的苯硼酸功能化高分 子纳米载体
}

\author{
赵丽 ${ }^{1,3 \dagger}$, 黄琼卫 ${ }^{2 \dagger}$, 王立艳 ${ }^{1}$, 盖广清 ${ }^{*}$, 肖姗姗 ${ }^{1}$, 毕菲 ${ }^{1}, 丁$ 建勋 ${ }^{3 *}$ \\ 1. 吉林建筑大学材料科学与工程学院, 长春 130118 \\ 2. 吉林大学中日联谊医院内分泌科, 长春 130033 \\ 3. 中国科学院长春应用化学研究所, 生态环境高分子材料重点实验室, 长春 130022 \\ $\dagger$ 同等贡献 \\ *通讯作者, E-mail: gaigq@163.com; jxding@ciac.ac.cn \\ 收稿日期: 2017-02-09; 接受日期: 2017-03-30; 网络版发表日期: 2017-08-28 \\ 国家自然科学基金(编号: 51403075, 51673190)和吉林省科技发展计划(编号: 20170520153JH, 20160204015SF)资助项目
}

\begin{abstract}
摘要近年来, 葡萄糖敏感高分子药物传递系统因其能够依据葡萄糖浓度变化自动连续地控制药物释放 而备受关注. 其中, 基于苯硼酸(PBA)的葡萄糖敏感高分子载体以其较好的稳定性、长期储存性和可逆的葡 萄糖敏感性能而被广泛研究, 特别是基于PBA的葡萄糖敏感纳米药物传输体系成为该领域的研究热点. 本文 系统地概述了用于自调式药物传输的葡萄糖敏感PBA基高分子纳米药物载体, 包括纳米胶束、纳米囊泡、 纳米凝胶以及杂化纳米粒子等的研究进展, 并对其发展方向和应用前景进行了展望.
\end{abstract}

关键词葡萄糖敏感, 苯嗍酸, 高分子, 纳米载体, 药物传递, 糖尿病治疗

\section{1 引言}

糖尿病是一种内源性疾病, 是由遗传和环境等因 素引起的胰岛功能衰退, 伴随以糖代谢紊乱为主要表 现症状的临床综合征. 它是一种慢性代谢性疾病, 与 心脑血管疾病和癌症并称为人类健康的“三大杀手”. 糖尿病的患病率逐年增长, 并呈现出年轻化的趋势, 严 重危害人类的生命健康 ${ }^{[1 \sim 3]}$. 世界卫生组织将糖尿病防 治列为全球保护人类健康的重要问题之一 ${ }^{[4]}$. 因此, 对 糖尿病的防治已经成为一个重大的医学和社会问题, 也是目前具有重大意义的研究课题之一. 糖尿病因其 病理学特点可以分为I型糖尿病和II型糖尿病 ${ }^{[5]}$. I 型糖
尿病即胰岛素依赖型糖尿病,需终身使用外来胰岛素 治疗. I型糖尿病约占糖尿病患者总数的 $10 \%$. II型糖 尿病即非胰岛素依赖型糖尿病, 是由胰岛 $\beta$ 细胞分泌 功能缺陷导致的胰岛素相对不足而血糖升高的症状. II 型糖尿病约占糖尿病总人数的 $90 \%$. 目前, 糖尿病治 疗最有效的途径是补充外源性胰岛素. 但胰岛素具有 分子量大、易变性以及脂溶性差等缺点而导致其口 服时的生物利用度低, 故对糖尿病的治疗仍采用常规 的注射给药方式. 虽然皮下注射胰岛素能够快速降低 血糖, 但每日数次的注射带给患者极大的身体和精神 痛苦, 因此长期注射胰岛素控制血糖时, 病人的耐受 性和顺从性较差. 虽然口服给药、鼻腔给药及超声介

引用格式: 赵丽, 黄琼卫, 王立艳, 盖广清, 肖姗姗, 毕菲, 丁建勋. 用于葡萄糖敏感药物释放的苯硼酸功能化高分子纳米载体. 中国科学: 化学, 2017, 47: 1057-1074

Zhao L, Huang Q, Wang L, Gai G, Xiao S, Bi F, Ding J. Phenylboronic acid-functionalized polymer nanocarriers for glucose-triggered drug release. Sci Sin Chim, 2017, 47: 1057-1074, doi: 10.1360/N032017-00021 
导给药等非注射胰岛素给药途径取得了较大的进展, 但因胰岛素的生物利用度很低且受制药技术的制约, 这些给药途径仍处于实验研究阶段 ${ }^{[6-9]}$.

近年来, 葡萄糖敏感药物传递系统因其可以依据 葡萄糖浓度的变化而自动连续地控制药物的释放而 备受关注, 有望取代常规注射给药途径, 成为一种潜 在的治疗方法 ${ }^{[10]}$. 葡萄糖敏感药物传递系统能够实时 监控血糖变化并调控胰岛素的释放, 从而实时控制血 糖水平, 更好地模拟人体胰腺分泌胰岛素的生理模式, 被称为“人工胰腺”. 该系统是一种自调式的药物控制 释放系统, 用于糖尿病的治疗不仅可以减少胰岛素的 注射次数更提高了患者的顺从性, 具有较大的糖尿病 治疗应用前景. 基于苯嗍酸(PBA)的葡萄糖敏感高分 子是一种全合成体系, 用于药物的自调式控制释放, 具有体系稳定、长期保存以及可逆葡萄糖响应的优 势, 成为近年来的研究热点之一 ${ }^{[11,12]}$.

PBA是一种路易斯酸, 其 $\mathrm{p} K_{\mathrm{a}}$ 值的范围在 8.2 8. $6^{[13]}$. PBA在药物传递领域具有极其重要的应用 ${ }^{[14 \sim 16]}$. 在水 溶液中PBA有两种存在形式,一种是未电离的平面三 角形的疏水形式, 另一种是电离的正四面体构型的亲 水形式. 在这两种形式的PBA间存在着可逆平衡 ${ }^{[17]}$. 两种形式的PBA都能与葡萄糖等 1,2 -二元醇或多元醇 特异性结合形成苯嗍酸酯复合物, 但电离形式的亲水 性PBA与葡萄糖形成的苯硼酸酯更稳定(图1). 当体系 的 $\mathrm{pH}$ 大于 $\mathrm{PBA}$ 的 $\mathrm{p} K_{\mathrm{a}}$ 时, $\mathrm{PBA}$ 功能化的载体与葡萄糖 特异性结合形成亲水性的苯硼酸酯. 苯硼酸酯的形成 使得PBA的电离平衡向正方向移动, 从而增加了电离 形式PBA的含量, 进而使电离形式的苯硼酸酯复合物 增加. 这样一方面提高了 PBA功能化载体材料的亲水 性, 另一方面由于平面三角形的PBA转化为正四面体 结构, 增大了载体材料的空间体积. 两种作用均使所 担载的药物响应于葡萄糖浓度的变化而释放出来, 且 药物释放速度和释放量随葡萄糖浓度的升高而增大. 这种特性赋予PBA功能化药物载体良好的葡萄糖敏 感性能. 这种基于PBA及其衍生物与 1,2 -二元醇或多 元醇间的特异性结合成为构筑葡萄糖敏感PBA基药 物传输体系的基础, 并在葡萄糖敏感药物传递系统具 有潜在的应用前景 ${ }^{[18,19]}$.

PBA及其衍生物用于糖尿病治疗的葡萄糖敏感药 物载体的前提是降低其 $\mathrm{p} K_{\mathrm{a}}$, 使其在人体生理 $\mathrm{pH}$ 下具 有葡萄糖敏感性能 ${ }^{[20]}$. 为此, 研究者采取了各种途径



图 1 PBA与葡萄糖的相互作用示意图(网络版彩图)

如在PBA上引入羧基 ${ }^{[13]}$ 、卤素或硝基 ${ }^{[21,22]}$ 等来提高 PBA在生理条件下的葡萄糖敏感性能. 此外, PBA功 能化的高分子材料用于葡萄糖敏感药物传递系统有 多种载体形式, 如水凝胶、微凝胶、微囊以及纳米载 体等 ${ }^{[16,23225]}$. 南开大学的史林启课题组 ${ }^{[26]}$ 对PBA基葡 萄糖响应性聚合物材料的胰岛素投递和血糖检测的 应用进行了综述, 介绍了共价键交联微/纳米凝胶、自 组装胶束和囊泡、模板法制备囊泡的方法及其葡萄 糖响应性能, 以及荧光感应器和比色典感应器对葡萄 糖浓度的检测研究. 该课题组还对含PBA材料在糖尿 病和癌症治疗相关的药物投递研究进行了综述 ${ }^{[27]}$. 然 而全面介绍PBA基纳米载体实现葡萄糖触发药物释 放的报道较少. 纳米药物载体因其特殊的尺寸效应在 药物传递领域具有更好的应用. PBA基葡萄糖敏感纳 米药物载体形式有纳米胶束、纳米囊泡、纳米凝胶 和杂化纳米粒子等. 本文重点介绍了基于PBA的葡萄 糖敏感高分子纳米药物载体的制备、性能和应用, 并 对该领域的发展前景进行了展望.

\section{2 葡萄糖敏感PBA基高分子纳米药物载体}

纳米药物载体因其独特的纳米特性而赋予载体 材料良好的性能, 如靶向性、较长的血液循环时间、 高载药率等. PBA功能化的高分子用于纳米药物载体 具有良好的葡萄糖敏感性能. 葡萄糖敏感药物传递系 统有望取代常规注射给药途径而成为一种潜在的糖 尿病治疗方法. 基于PBA的纳米药物载体主要有纳米 胶束、纳米囊泡、纳米凝胶和杂化纳米粒子等(图2). 近年来, 随着研究者的不断努力和探索，葡萄糖敏感 


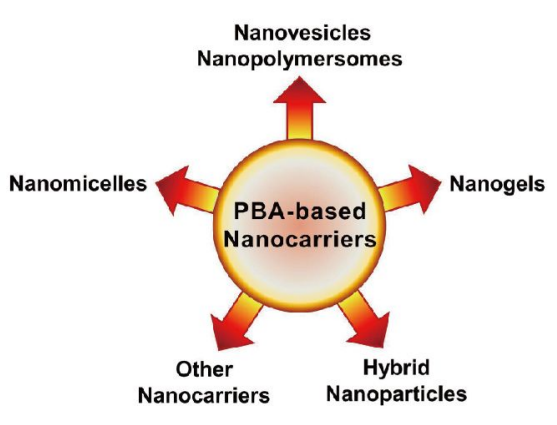

图 2 基于PBA的高分子纳米药物载体(网络版彩图)

$\mathrm{PBA}$ 基高分子纳米药物载体取得了较大的进展.

\subsection{PBA基纳米胶束体系}

聚合物胶束是由两亲性聚合物在溶液中自组装 而得到的具有核-壳结构的聚集体. 两亲性的共聚物 的亲水段多为聚乙二醇(PEG), 在水溶液中形成胶束 的外壳, 提供必要的亲水性并稳定内核. 疏水段可 由多种材料组成, 并在水溶液中形成胶束的内核. 这 种核-壳结构赋予聚合物胶束良好的性能, 如较高的 载药量和载药效率、较长的体内循环时间、可修饰 性和较高的生物利用度等 ${ }^{[28]}$. PBA功能化的聚合物 胶束因其简单的制备方法和较好的葡萄糖敏感性能 引起研究者极大的兴趣, 对其的研究也取得较大的 进展.

采用可逆加成-断裂转移自由基聚合(RAFT)可以

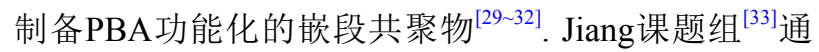
过分子设计, 采用RAFT聚合方法, 以聚(甲氧基聚乙二 醇丙烯酰胺)为大分子引发剂(MePEGA)引发3-丙烯酰 胺基苯硼酸(AAPBA)和邻硝基茮基丙烯酸酯(NBA)共 聚制备了具有光和葡萄糖双敏感的聚合物纳米胶束. 以胰岛素为模型药物, 该课题组考察了该聚合物纳米 胶束的光和葡萄糖响应的胰岛素释放性能. 在可见光 照射下, $30 \mathrm{~min}$ 内只有 $20 \%$ 的胰岛素释放出来, 而在紫 外光照射下 $\left(\lambda_{\max }=365 \mathrm{~nm}, 75 \mathrm{~mW} \mathrm{~cm}{ }^{-2}\right), 30 \mathrm{~min}$ 内胰岛 素的累积释放量达到了 $98 \%$. 这是因为在紫外光照射 下疏水的聚邻硝基苯甲基丙烯酸酯(PNBA)转变成亲 水性的聚丙烯酸, 聚合物胶束的亲疏水平衡向亲水性 方向转移, 破坏了胶束的原有结构并促使胰岛素快速 释放. 该聚合物胶束调控胰岛素的释放特性也具有葡 萄糖响应性. 在不含葡萄糖的溶液中, $24 \mathrm{~h}$ 内胰岛素的 累积释放量只有 $5 \%$, 几乎可以省略. 而在葡萄糖溶液
中时, 胰岛素的累积释放量急剧增加. 当葡萄糖浓度 为 $25 \mathrm{mM}$ 时, $24 \mathrm{~h}$ 内胰岛素的累积释放量达到了 $93 \%$. 甲基噻唑蓝(MTT)检测证实, 该纳米胶束具有较好的 生物相容性, 圆二色谱 $(\mathrm{CD})$ 检测结果表明, 所释放出 的胰岛素仍具有其原有的二级结构和活性. 这种紫外 光和葡萄糖双敏感的聚合物纳米胶束在胰岛素自调 式控制释放中具有潜在的应用前景. 同样采用RAFT 聚合方法, 该课题组结合酶法酯交换技术, 一步制备 了具有苂光性能的葡萄糖敏感纳米胶束, 为聚合物胶 束的制备提供了新方法 ${ }^{[34]}$.

除了RAFT 聚合方法外, 采用原子转移自由基 聚合(ATRP) 也是制备葡萄糖敏感嵌段共聚物的有 效方法 ${ }^{[35]}$. Yang 课题组 ${ }^{[36]}$ 采用ATRP聚合方法, 以 mPEG5000-Br 为大分子引发剂引发自制单体(2-苯嗍 酸酯-1,3-二恶烷-5-乙基)丙烯酸甲基酯(PBDEMA)聚 合制备了嵌段共聚物 mPEG5000- $b$-PPBDEMA且该嵌 段共聚物能自组装成纳米胶束. 如图3所示, 该两亲性 嵌段共聚物侧链上的苯硼酸酯基团在遇到葡萄糖分 子时可断裂, 使两亲性的共聚物转变成双亲性的共聚 物, 从而使聚合物纳米胶束的亲水性增加, 体积增大甚 至解体. 这种胶束在 $\mathrm{pH} 7.4$ 时具有非常快速的葡萄糖 响应药物释放特性, 所担载的疏水药物罗丹明在 $3 \mathrm{~min}$ 内的累积释放量高达 $40 \%$, 而担载的胰岛素也能响应 葡萄糖而快速释放出来, 具有“开-关”式的释放特性.

为了得到转变 $\mathrm{pH}$ 合适的 $\mathrm{PBA}$ 衍生物以用作葡萄糖 敏感药物传输载体, 许多研究者进行了大量研究. Shi课 题组 ${ }^{[37]}$ 采用ATRP引发剂PEG-Br引发丙烯酸叔丁酯聚 合, 并通过脱除叔丁基保护和进一步经3-氨基苯䃉酸修 饰, 最终得到嵌段共聚物聚(乙二醇)-block-聚(丙烯酸$c o$-丙烯酰胺基苯硼酸) (PEG- $b$-P(AA- $c o-A A P B A))$. 该 嵌段共聚物自组装成以 $\mathrm{P}(\mathrm{AA}-\mathrm{A}-\mathrm{A}-\mathrm{APBA})$ 为内核, 以亲 水性PEG为外壳的纳米胶束. 由于羧基和嗍原子的配 位作用能够降低PBA的 $\mathrm{p} K_{\mathrm{a}}$, 所以通过控制PBA的修饰 率可有效调节聚合物胶束呈现葡萄糖敏感性能的 $\mathrm{pH}$ 范 围. 当PBA的修饰率为 $63 \%$ 时, 可得到生理 $\mathrm{pH}$ 条件下具 有良好葡萄糖敏感性能的纳米胶束. 除此之外, Shi课 题组 ${ }^{[38,39]}$ 还制备了葡萄糖敏感性能增强的PBA-糖聚物 纳米复合胶束体系. 其中糖基化聚合物 $\mathrm{P}(\mathrm{AA}-\mathrm{co}-\mathrm{AGA})$ 是聚丙烯酸接枝2-氨基葡萄糖得到的. 该糖基化聚合 物与 PBA功能化的嵌段共聚物PEG- $b$-P(AA-co-APBA) 复合得到以 $\mathrm{P}(\mathrm{AA}-c o-\mathrm{APBA}) / \mathrm{P}(\mathrm{AA}-c o-\mathrm{AGA})$ 为内核、 


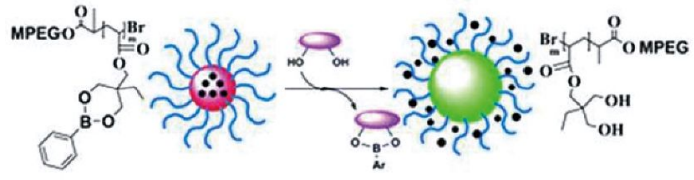

图 3 聚合物胶束的葡萄糖敏感性能示意图 ${ }^{[36]}$ (网络版 彩图)

以 PEG为外壳的纳米复合胶束. 与单一胶束相比, 该 纳米复合胶束具有更好的稳定性和生物相容性, 并在 生理条件下在 $2.0 \mathrm{mg} \mathrm{mL}^{-1}$ 的葡萄糖溶液中(模拟糖尿 病患者的餐后血糖值)即可表现出较好的葡萄糖敏感 性能, 为葡萄糖敏感聚合物胶束的构筑提供了一种有 效的方法. 除嵌段共聚物外, PBA功能化的两亲性接 枝共聚物也能自组装成葡萄糖敏感的聚合物胶束 ${ }^{[40]}$.

采用丙烯酸类单体制备葡萄糖敏感的聚合物胶 束快速有效且制备的聚合物胶束具有较好的生物相 容性和葡萄糖敏感性能, 但聚丙烯酸类材料的不可生 物降解性限制了其临床应用. 聚氨基酸具有良好的生 物相容性和可生物降解性, 被广泛应用于药物传递、 基因治疗等生物医学领域 ${ }^{[41-44]}$. 葡萄糖敏感的聚氨基 酸纳米粒子的研究也取得较大的进展. 本课题组通过 $\gamma$-苯甲基- $L$-谷氨酸酯- $N$-内羧酸䣶(BLG-NCA) 开环聚 合, 脱掉苯甲基保护和3-氨基苯硼酸修饰得到聚(乙二 醇单甲醚)-block-聚 ( $L$-谷氨酸- $c o$ - 谷氨酰胺基苯硼酸) (mPEG- $b-\mathrm{P}(\mathrm{GA}-c o-\mathrm{GPBA})$ ) 嵌段共聚物, 并对其自组装 成的纳米胶束在生理 $\mathrm{pH}$ 下的胰岛素自调式控制释放 行为进行了研究 ${ }^{[45]}$. MTT检测和兔溶血试验表明, 该 聚氨基酸纳米胶束具有良好的生物相容性. 该纳米 胶束能够依据葡萄糖浓度的变化调节胰岛素的释放 行为, 使胰岛素的释放具有随葡萄糖浓度变化而呈现 “开-关”式释放的特性, 且释放出的胰岛素具有其原有 的活性. 该聚氨基酸纳米胶束体系较好地克服了药物 担载和释放中药物活性易于失活的问题, 具有体内按 需“开-关”式释放药物的潜力. 此外, 聚氨基酸类载体 材料也保证该药物传递体系具有良好的生物相容性 和可生物降解性能.

同样采用开环聚合, Shi课题组 ${ }^{[46]}$ 制备了聚乙二 醇-block-聚(天冬氨酸- $c o$-天冬酰胺基苯硼酸) (PEG- $b$ $\mathrm{P}($ Asp-co-AspPBA)) 和聚异丙基丙烯酰胺-block-聚(天 冬氨酸- $c o$-天冬酰胺基苯嗍酸) (PNIPAM- $b$-P(Asp- $c o$ AspPBA)) 嵌段共聚物. 这两种聚氨基酸嵌段共聚物
能够自组装成聚合物复合胶束. 这种纳米复合胶束具 有P(Asp-co-AspPBA)疏水内核和混合的PEG/PNIPAM 外壳. 当温度升高至 $37^{\circ} \mathrm{C}$ 后, PNIPAM会塌缩在复合胶 束内核的表面形成一层连续的膜结构,并在中间嵌有 PEG层, 使复合胶束的核-壳结构转变为核-壳-冠结构. 此时复合胶束展现出可逆的葡萄糖响应溶胀行为, 并 能反复“开-关”式释放胰岛素. 另外, 连续的PNIPAM 膜还可以保护被包覆的胰岛素免受蛋白酶的降解,使 其具有原有的生物活性. 该药物传递体系的巧妙设计, 不仅使纳米载体具有较好的“开-关”式按需释放药物 的特性, 保证了药物原有的活性, 也具有较好的生物 相容性和可生物降解性能. 这种“开-关”式胰岛素自 调式控制释放特性和保护药物的作用, 使得该葡萄糖 敏感纳米复合胶束在糖尿病治疗方面具有较大的潜 在应用。

两亲性的嵌段共聚物在溶液中不仅能自组装成 纳米胶束, 也可以自组装成交联纳米胶束. PBA功能 化的聚多糖自组装成的交联胶束因其聚合物分子内 PBA基团与糖单元上二元醇间的自交联作用, 比纳米 胶束更稳定.

$\mathrm{Li}$ 等 ${ }^{[47]}$ 采用 $\mathrm{RAFT}$ 聚合方法和类糖单体甲基丙 烯酸 2-( $N$ - 葡萄糖酰胺) 乙酯 (GAMA) 以及 AAPBA制 备了 PBA功能化的含糖共聚物 P(AAPBA- $b$-GAMA) (图4(a)). 聚合物分子上的PBA基团与碳水化合物上的 二羟基特异性结合而促使共聚物自组装成交联纳米 粒子(图4(b)). 该交联纳米粒子具有亲水性的PGAMA 冠和疏水的PAAPBA核. PBA赋予嵌段共聚物良好的 葡萄糖敏感性能, 而GAMA作为一种新型的可再生糖, 不仅可以降低PBA的 $\mathrm{p} K_{\mathrm{a}}$, 还可以降低其生物毒性. 当 有葡萄糖小分子存在时, 游离的葡萄糖将与类糖碳水 化合物竞争性地与PBA结合, 从而使纳米粒子疏松甚 至解体. 该糖基化聚合物纳米粒子在生理 $\mathrm{pH}$ 时可响应 血糖浓度的变化而调控胰岛素的释放(图4(c)), 且这种 葡萄糖敏感药物控制释放具有可逆性, 从而避免血糖 波动并减少并发症. 这种智能药物输送系统在糖尿病 治疗中具有潜在的应用前景。

在前期工作的基础上, Li课题组 ${ }^{[48]}$ 制备了甲基丙 烯酸2-( $N$-葡萄糖酰胺)乙酯(GAMA)和AAPBA无规共 聚的糖基化聚合物 $\mathrm{P}(\mathrm{GAMA} a-r-\mathrm{AAPBA} b)$, 其中 $a$ 和 $b$ 是单体GAMA和AAPBA的摩尔投料比. 该两亲性无 规共聚物自组装成的纳米粒子用于胰岛素的担载具有 
(a)<smiles>C=CC(=O)Nc1cccc(B(O)O)c1</smiles>
AAPBA DMP

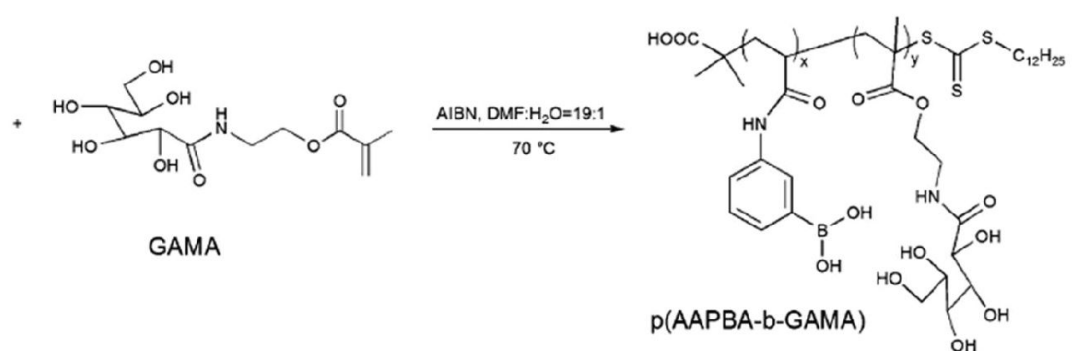

(b)



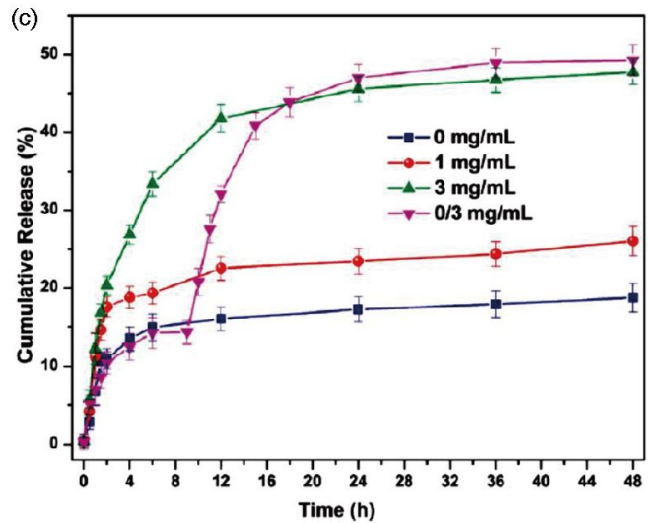

图 4 (a) P(AAPBA- $b$-GAMA)的合成; (b) P(AAPBA- $b$-GAMA)纳米粒子示意图及纳米粒子在 $\mathrm{pH}$ 7.4的磷酸盐缓冲液(PBS)中 不同葡萄糖浓度下的胰岛素释放; (c) P(AAPBA- $b$-GAMA)纳米粒子在无糖介质中释放 $9 \mathrm{~h}$ 后转入 $3 \mathrm{mg} \mathrm{mL}^{-1}$ 葡萄糖中的胰岛 素释放曲线 ${ }^{[47]}$ (网络版彩图)

$11.2 \%$ 的载药量和 $81.9 \%$ 的包封率, 为其良好的糖尿病 大鼠降血糖效果奠定了基础. 该纳米粒子具有在糖尿 病患者血糖水平下快速释放胰岛素而在正常血糖水 平时缓慢释放胰岛素的特性, 且纳米粒子中AAPBA含 量越高, 胰岛素的葡萄糖响应释放性能越明显(图5). 2-脱氧葡萄糖摄取实验表明, 胰岛素和载胰岛素纳米 粒子具有相同的生理功能. 此外, Western blot分析和 免疫荧光实验证实, 该载药纳米粒子所释放出的胰岛 素与常规胰岛素具有相同的降糖机制. 最重要的是, 体内实验表明, 该纳米粒子具有良好的降血糖效果. 如表1所示, 对糖尿病大鼠口服载胰岛素纳米粒子后, 血浆葡萄糖水平降低总量 $(D \%)$ 和药理相对生物利用 度 $(F \%)$ 均有所提高, 进一步证实该纳米粒子具有葡萄
糖响应多肽和蛋白质传递的应用潜力. Wang 等 ${ }^{[49]}$ 采 用GAMA和甲基丙烯酰胺基苯嗍酸(MAPBA)为单体 制备了直径为 $120 \mathrm{~nm}$ 的P(GAMA- $r$-MAPBA)纳米粒子, 该纳米粒子具有较好的球形形貌和葡萄糖响应胰岛 素释放性能.

同样采用嵌段共聚物分子内自交联的方式, $\mathrm{Li}$ 课题组 ${ }^{[50]}$ 成功制备了 PBA 修饰的含糖聚合物聚(丙 烯酸- $c o$-丙烯酰胺基苯砋酸)-block-聚(2-丙烯酰基氧 乙基半乳糖)-block-聚(丙烯酸- $c o$-丙烯酰胺基苯硼酸) (PAA-co-PAAPBA)- $b-)_{2}$ PAEG), 并自组装成交联纳米胶 束. 含糖聚合物可有效降低PBA的毒性, 赋予纳米粒 子良好的生物相容性. 此外, 该交联纳米胶束还具 有良好的 $\mathrm{pH}$ 和葡萄糖双敏感胰岛素释放行为. Cheng 

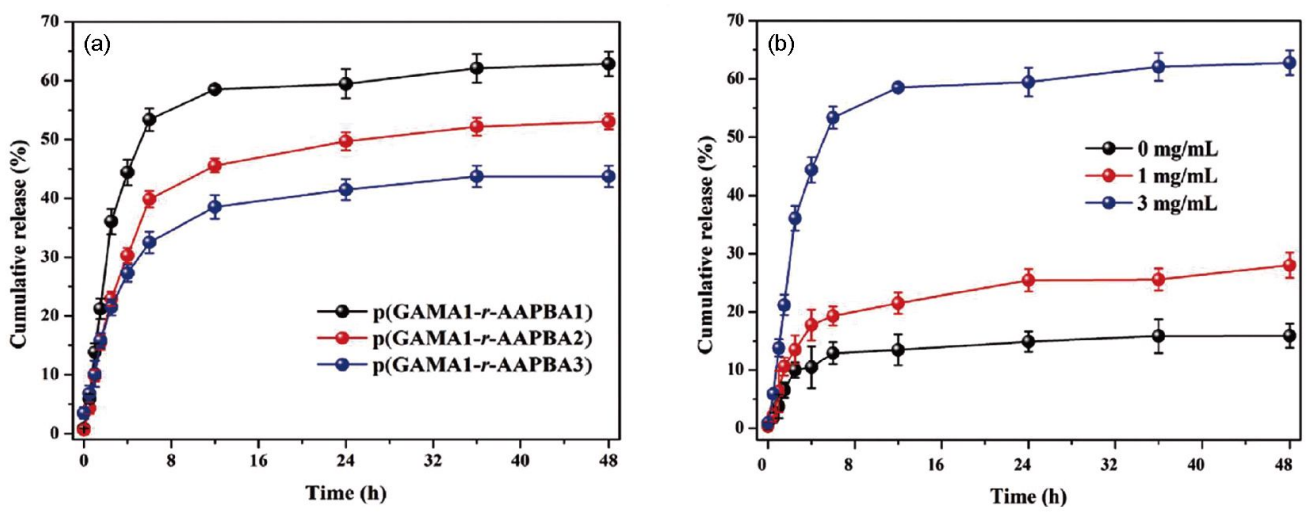

图 5 (a) $\mathrm{P}\left(\mathrm{GAMA}-r\right.$-AAPBA) 纳米粒子在 $\mathrm{pH} 7.4$ 含 $3 \mathrm{mg} \mathrm{mL}^{-1}$ 葡萄糖PBS中的胰岛素累积释放曲线; (b) P(GAMA1-r-AAPBA1) 纳米粒子在不同葡萄糖浓度溶液中的胰岛素累积释放曲线 ${ }^{[48]}$ (网络版彩图)

表 1 口服P(GAMA1-r-AAPBA2)纳米粒子悬浊液给药(胰岛素剂量10、20、40 $\mathrm{IU} \mathrm{kg}^{-1}$ )的药代动力学参数 ${ }^{[8]}$

\begin{tabular}{ccccc}
\hline 样品 & $T_{\min }{ }^{\text {a) }}(\mathrm{h})$ & $\begin{array}{c}C_{\min }{ }^{\mathrm{b})}(\%, \text { 占初始葡萄 } \\
\text { 糖水平的百分比 })\end{array}$ & $D^{\mathrm{c}}$ & $F^{\mathrm{d}}$ \\
\hline 皮下注射胰岛素(insulin) & 0.5 & 45.8 & $8.89 \pm 1.7$ & $\sim 100$ \\
insulin+PBS & 0.5 & 96.6 & $0.525 \pm 1.7$ & $0.590 \pm 1.2$ \\
$10 \mathrm{IU} \mathrm{kg}^{-1}$ & 2 & 59.6 & $19.2 \pm 6.3$ & $21.4 \pm 3.4$ \\
$20 \mathrm{IU} \mathrm{kg}^{-1}$ & 4 & 30.8 & $40.7 \pm 5.2$ & $45.8 \pm 3.1$ \\
$40 \mathrm{IU} \mathrm{kg}^{-1}$ & 4 & 18.0 & $46.8 \pm 5.6$ & $52.6 \pm 2.8$ \\
\hline
\end{tabular}

a) $T_{\min }$ 代表出现最低葡萄糖水平时的时间; b) $C_{\min }$ 代表最低葡萄糖水平占初始葡萄糖水平的百分比; c) $D \%$ 代表血浆葡萄糖水平降 低总量; d) $F \%$ 为药理相对生物利用度.

等 ${ }^{[51]}$ 研究了一种通过RAFT聚合得到的新型两亲性嵌 段共聚物聚(甲基丙烯酸-2-( $N$-乳糖酰胺)乙酯)-block聚(3-丙烯酰胺基苯硼酸) (P(LAMA- $b$-AAPBA)), 并能 够自组装成平均尺寸为 $238 \sim 403 \mathrm{~nm}$ 的球形粒子. 该纳 米粒子用于胰岛素的担载具有良好的葡萄糖响应药 物释放性能. 此外, 该纳米粒子还具有较好的生物相 容性, 是一种较有前途的葡萄糖敏感载体材料.

$\mathrm{Li}^{\text {等 }}{ }^{[52]}$ 采用AAPBA和2-(丙烯酰胺)吡喃葡萄糖 (AGA)单体通过RAFT聚合制备了嵌段和无规共聚物. 两种PBA功能化的聚多糖都能发生分子内交联, 形成 葡萄糖敏感的聚合物纳米粒子(图6(A)). 两种纳米粒 子用于胰岛素的担载均具有较好的葡萄糖敏感药物 释放性能, 但无规交替聚合物纳米粒子具有更明显 的葡萄糖响应胰岛素释放特性(图6(B, C)), 表明聚多 糖的结构对纳米粒子的自组装性能和葡萄糖敏感性 能具有重要的影响. 此外, 采用AAPBA和马来酰亚胺 葡萄糖胺 (MAGA) 的共聚物P(AAPBA- $r$-MAGA) 以及
PBA接枝壳聚糖(PBACS), 也可用于葡萄糖敏感纳米 粒子的组装 ${ }^{[3,54]}$. Herrera-Alonso等 ${ }^{[5]}$ 采用PEG大分子 引发剂引发含PBA的环酯开环聚合得到有机硼共聚 物 mPEG- $b-P P B C$ (图7). 该共聚物能够依据聚合物的 亲疏水比例自组装成球形的纳米粒子和类似囊泡的 聚集体, 两者均具有良好的葡萄糖敏感性能.

除采用PBA功能化的聚多糖自交联作用形成交 联纳米胶束外, PBA功能化聚合物上的PBA基团和聚 多糖分子上二元醇发生特异性结合作用也能形成纳 米胶束. 聚(3-甲基丙烯酰胺基苯硼酸) (PMAPBA)与 葡聚糖(Dex)上的糖单元发生络合作用形成葡萄糖敏 感的纳米粒子 ${ }^{[56]}$. PMAPBA上的PBA也可以与壳聚糖 上的氨基相互作用形成纳米粒子 ${ }^{[57]}$.

\subsection{PBA基纳米囊泡体系}

PBA 功能化的嵌段共聚物不仅可以自组装形成 葡萄糖敏感的纳米胶束和交联纳米胶束的纳米粒子结 
(A)

(a) Yr?

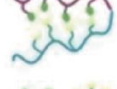

inter- and intramolecular self-assembly

reks interactions interactions
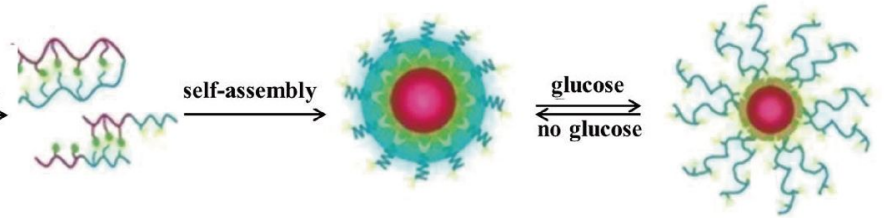

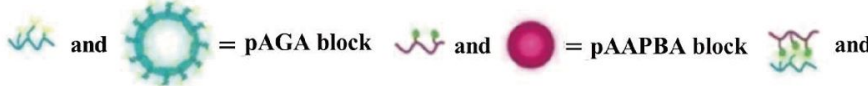

inter- and intramolecular

Complexation between

AGA and AAPBA

(b)
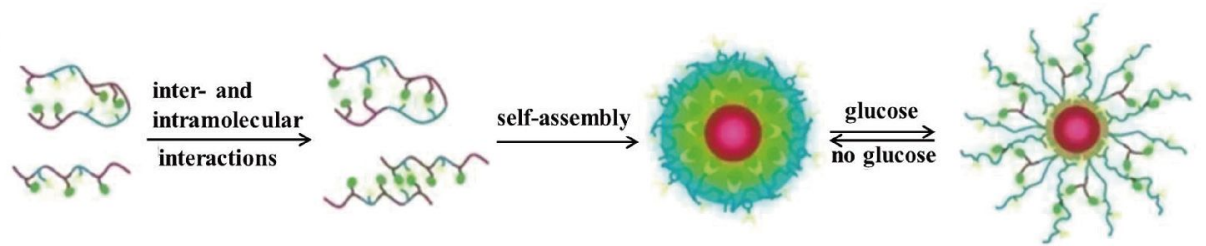

$\checkmark$ and

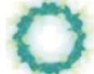

AGA moieties $h$ and

$=$ AAPBA moieties

(2) inter- and intramolecular

AGA and AAPBA


图 6 (A) 嵌段聚多糖(a)和无规聚多糖(b)自组装成纳米粒子的示意图; 嵌段聚多糖(B)和无规聚多糖(C)在 $\mathrm{pH} 7.4$ 含 $3 \mathrm{mg} \mathrm{mL}^{-1}$ 葡 萄糖PBS中的胰岛素的累积释放曲线 ${ }^{[52]}$ (网络版彩图)

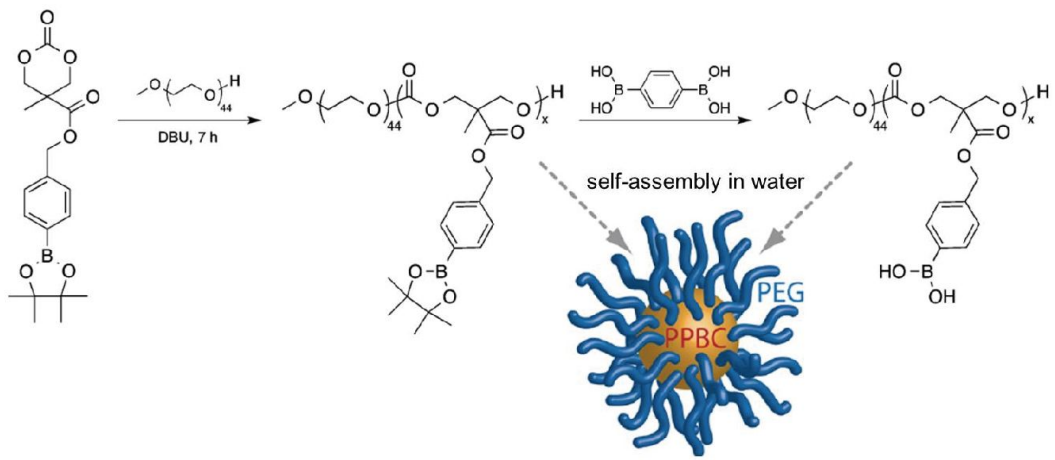

图 7 有机嗍聚合物的合成和纳米粒子形成示意图 ${ }^{[55]}$ (网络版彩图)

构, 也能自组装成葡萄糖敏感的聚合物囊泡. 两亲性 嵌段共聚物自组装纳米粒子的形貌主要取决于嵌段 共聚物的结构. 当两亲性嵌段共聚物的亲水段所占聚
合物分子比例较大时, 共聚物易于自组装成聚合物胶 束. 当聚合物疏水段所占比例较大时(疏水段质量为 聚合物分子质量的40\% 80\%), 嵌段共聚物在溶液中 
易形成囊泡结构, 且囊泡的大小受聚合物的浓度、选 择性溶剂的用量以及溶液的 $\mathrm{pH}$ 等因素的影响. 当聚合 物疏水段所占比例更大时(疏水段质量与聚合物分子 质量比> 60\% 80\%), 嵌段共聚物在溶液中易形成紧密 纳米粒子 ${ }^{[8]}$. 由密闭双分子层所形成的球形或椭球形 中空结构使聚合物囊泡在药物传递领域具有重要应 用. 与聚合物胶束相比, 聚合物囊泡的中空结构赋予 其更高的载药量. 葡萄糖敏感的聚合物囊泡因其能提 高药物的担载量和生物活性而受到广泛关注.

Yang课题组 ${ }^{[36]}$ 采用ATRP聚合方法得到嵌段共聚 物mPEG5000- $b$-PPBDEMA, 该共聚物在水溶液中自组 装成的纳米胶束具有良好的葡萄糖敏感性能. 除此之 外, 该两亲性嵌段共聚物还能够组装成纳米囊泡 ${ }^{[59]}$. 通 过中子小角散射实验, 该课题组证实PEG与质量分数 为 $24 \%$ 的嵌段共聚物 mPEG5000- $b$-PPBDEMA在质量 分数为 $0.1 \%$ 和 $0.5 \%$ 的葡萄糖溶液中时能够自组装成 囊泡. 在低葡萄糖浓度时该纳米囊泡能够相对稳定存 在, 而在高葡萄糖浓度时, 由于葡萄糖分子与PBDEMA 的相互作用增强该囊泡的结构稳定性减弱. 此外, 溶 液的 $\mathrm{pH}$ 也会影响该嵌段共聚物在溶液中的形貌.

van Hest 等 ${ }^{[60]}$ 报道了一种葡萄糖敏感聚合物囊 泡. 该囊泡由两种嵌段共聚物聚乙二醇-block-聚苯 乙烯硼酸 (PEG- $b$-PSBA) 和聚乙二醇-block-聚苯乙烯 (PEG- $b$-PS) 自组装而成. 当在葡萄糖溶液中时, 该囊 泡的疏水层变得疏松并产生很多小孔, 这主要是由于 PS和PSBA不混溶造成的. 在葡萄糖溶液中, 葡萄糖与 PBA上的嗍酸特异性结合形成苯矽酸酯, 增加了PSBA 的亲水性, 使其与PS的相容性变差并产生孔隙. 通过 改变两种嵌段共聚物可以调节囊泡疏水层上的孔隙 大小, 从而调节聚合物囊泡的膜渗透性. 相比于单一 共聚物PEG- $b$-PSBA形成的囊泡, 这种由两种嵌段共 聚物自组装成的囊泡具有更好的葡萄糖敏感可控药 物释放的特性. 同样采用乙烯基苯嗍酸衍生物乙烯 基苯硼氧化物(BOx), Kim 等 ${ }^{[61]}$ 通过RAFT聚合方法得 到嵌段共聚物PEG- $b$-PBOx 并自组装成纳米囊泡. 该 纳米囊泡能够在生理 $\mathrm{pH}$ 条件下响应单糖(葡萄糖或果 糖)而解体并释放出异硫氰酸荧光素(FITC)标记的胰 岛素, 而在没有单糖与聚合物囊泡中PBA基团相互作 用时则无FITC标记的胰岛素释放. 这种新型PBA共聚 物自组装成的纳米囊泡在传感器和药物传递领域具 有很好的应用潜能.
环糊精 $(C D)$, 通常被称为络合剂, 能与客体分子 通过分子间非共价键作用力形成主客体复合物, 在 药物传递领域中具有较多应用. Shi 等 ${ }^{[62]}$ 基于环糊 精主客体相互作用, 以其做模板制备了葡萄糖敏感 的聚合物囊泡。该课题组首先制备了含葡萄糖胺 的共聚物聚乙二醇-block - 聚( 天冬氨酸- $c 0$-天冬酰胺 基葡萄糖) ( $\left.\mathrm{PEG}_{45}-b-\mathrm{P}(\mathrm{Asp}-c o-\mathrm{AspGA})\right)$. 当加入 $\alpha$-环糊 精 $(\alpha-\mathrm{CD})$ 时，由于不溶性的 $\alpha-\mathrm{CD} / \mathrm{PEG}_{45}$ 包合物的生 成, $\mathrm{PEG}_{45}-b$-P(Asp-co-AspGA) 自组装成核-壳结构的 纳米胶束. 当向该胶束中加入 $\mathrm{PBA}$ 功能化的共聚物 $\mathrm{PEG}_{114}-b-\mathrm{P}(\mathrm{Asp}-\mathrm{co}-\mathrm{AspPBA})$ 时, 胶束的核-壳结构转变 为核-壳-冠结构. 其中, $\alpha-C D / \mathrm{PEG}_{45}$ 不溶物为核、 $\mathrm{PEG}_{114}$ 为冠. $\mathrm{P}(\mathrm{Asp}-c o-\mathrm{AspGA}) / \mathrm{P}(\mathrm{Asp}-c o-\mathrm{AspPBA})$ 则为中间 的壳层且壳层中有苯硼酸酯的交联结构, 其中苯硼 酸酯来源于 $\mathrm{GA}$ 和 PBA间的特异性结合. 当将 $\alpha-C D$ 移除时, 则得到内外层均由 PEG 组成、而中间层为 $\mathrm{P}(\mathrm{Asp}-\mathrm{co}-\mathrm{AspGA}) / \mathrm{P}(\mathrm{Asp}-\mathrm{co}-\mathrm{AspPBA})$ 结构的聚合物囊 泡且该囊泡具有较好的葡萄糖敏感药物释放特性. 此 外，该课题组 ${ }^{[63]}$ 还采用热敏感胶束为模板制备了葡 萄糖敏感的聚合物囊泡. 其中, 热敏感胶束的制备 是 PNIPAM- $b$-P(Asp-co-AspPBA) 在高于 PNIPAM的低 临界溶液温度(LCST)时自组装而成的, 其中PNIPAM 为核、 $\mathrm{P}(\mathrm{Asp}-c o-A s p P B A)$ 为壳. 当 $\mathrm{PEG}_{45}-b-\mathrm{P}(\mathrm{Asp}-c o-$ AspGA)加入热敏感聚合物胶束中时, 核-壳结构的纳米 胶束转变为核-壳-冠结构. 核-壳-冠结构的纳米胶束在 低于PNIPAM的LCST温度时, 形成具有膨胀的PNIPAM 内层、交联的 $\mathrm{P}(\mathrm{Asp}-c o-\mathrm{AspGA}) / \mathrm{P}(\mathrm{Asp}-c o-\mathrm{AspPBA})$ 膜 以及 $P E G$ 冠层结构的聚合物囊泡(图8).

Chen等 ${ }^{[64]}$ 制备了3-羧基苯嗍酸修饰的壳聚糖(CS$g$-CPBA), 并以 $\mathrm{SiO}_{2}$ 为模板将其与海藻酸钠 (Alg) 层层 自组装得到( $\left.\mathrm{Alg} / \mathrm{CS}-g-\mathrm{CPBA}_{m}\right)_{n}$ 囊泡. $\mathrm{Ca}^{2+}{ }^{2}$ 对 $\mathrm{Alg}$ 层具有 一定的鳌合作用(图9(A)). 该囊泡在 $\mathrm{Ca}^{2+}$ 螯合作用下能 够稳定存在, 且稳定性随着多层膜数量的增加而提高 (图9(B)). 在葡萄糖的作用下, 该纳米囊泡的尺寸增加

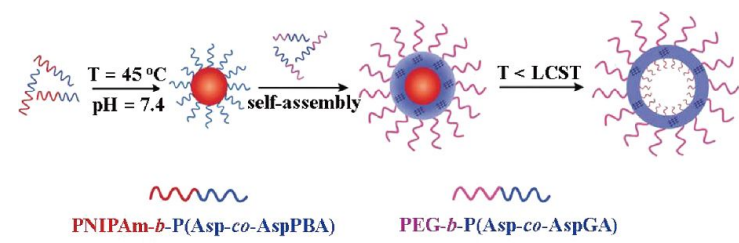

图 8 聚合物囊泡的形成示意图 ${ }^{[63]}$ (网络版彩图) 
(A)

(a)<smiles>CCCC1(C)CCCCC(C)(CC(C)(C)C)C1</smiles>

(b)

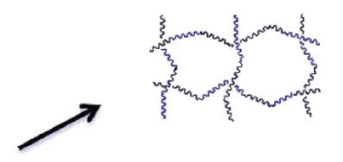
$\stackrel{1.200}{\stackrel{1}{1}, 3}$

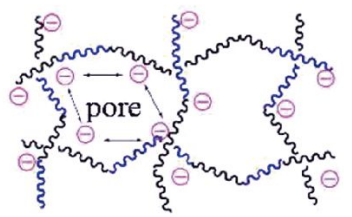

(c)


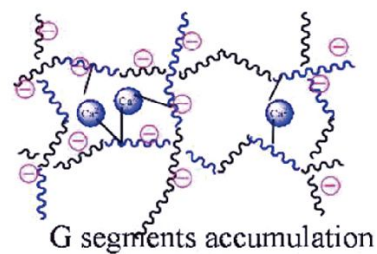
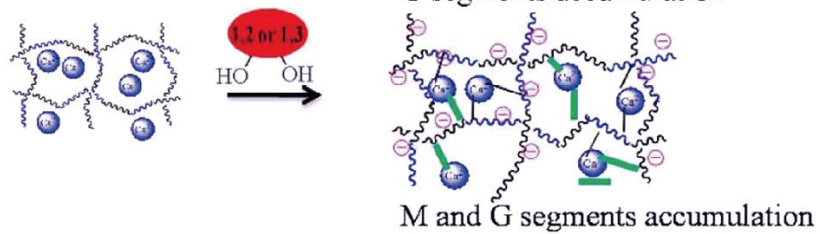



$\Theta$ : glucose response interaction - : chelation - : electrostatic interaction

(B)


(C)

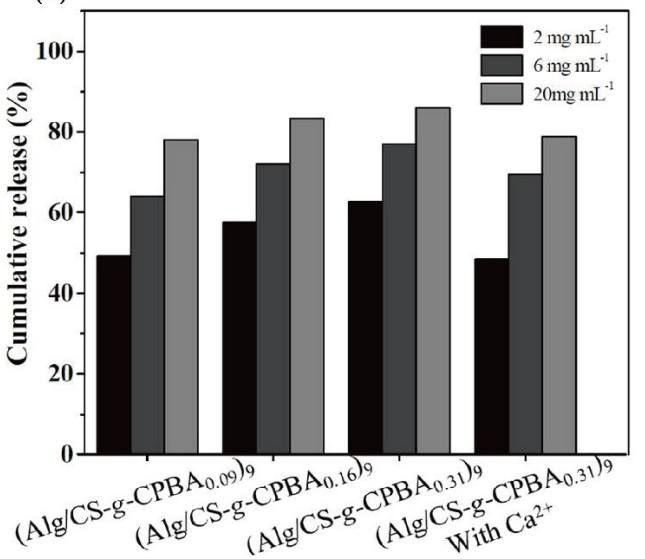

图 9 (A) 聚合物囊泡的形成示意图; (B) 无 $\mathrm{Ca}^{2+}$ 的 $\left(\mathrm{Alg} / \mathrm{CS}-\mathrm{g}-\mathrm{CPBA} \mathrm{PB}_{0.31}\right)_{5}(\mathrm{a})$ 和 $\left(\mathrm{Alg} / \mathrm{CS}-g-\mathrm{CPBA}_{0.31}\right)_{9}$ 囊泡(b), $\mathrm{Ca}^{2+}$ 含量为 $0.5 \%$ (c) 和 $1 \%$ (d)的 $\left(\mathrm{Alg} / \mathrm{CS}-\mathrm{g}-\mathrm{CPBA}_{0.31}\right)_{9}$ 囊泡的电子显微镜照片; (C) 不同囊泡在 $12 \mathrm{~h}$ 时胰岛素的累积释放百分数 ${ }^{[64]}$ (网络版彩图)

并发生溶胀而将所担载的胰岛素释放出来(图 $9(\mathrm{C}))$. 在 相同层数下, 该纳米囊泡的葡萄糖敏感胰岛素释放性 能随着壳聚糖上PBA含量的增加而增强. 虽然 $\mathrm{Ca}^{2+}$ 的 螯合作用能够显著提高纳米囊泡的稳定性, 但其葡萄 糖响应药物释放特性会有所减弱.

虽然葡萄糖敏感的聚合物囊泡因其中空结构用 于药物的传输具有更高的载药量, 并能保护药物的生 物活性, 但聚合物囊泡的制备方法与聚合物胶束相比 更为复杂, 在一定程度上限制了其应用.

\subsection{PBA 基纳米凝胶}

相比于纳米胶束的浓度依赖性解体和囊泡的尺 寸不易受控, 具有交联结构的纳米凝胶则更加稳定. 葡萄糖敏感纳米凝胶具有可逆的溶胀/收缩平衡, 赋予 其更好的葡萄糖敏感性能, 也得到广泛的关注. 当有 葡萄糖存在时, PBA修饰的纳米凝胶体积膨胀, 这主 要是由于葡萄糖与PBA结合形成的苯硼酸酯增加了 纳米凝胶的亲水性, 并促使其膨胀, 进而将所担载的 药物释放出来. 但目前基于PBA的葡萄糖敏感水凝胶 
和微凝胶的研究取得较大进展, 而对葡萄糖敏感纳米 凝胶的研究相对较少 ${ }^{[18,20,65 \sim 71]}$.

Zha课题组 ${ }^{[72]}$ 以 $\mathrm{SiO}_{2}$ 为模板, 采用模板法制备了含 有PNIPAM和聚 ( $N$-苯硼酸基丙烯酰胺) 的具有温度和 葡萄糖双重敏感性能的空心纳米凝胶. 以 $N, N$-亚甲基 双丙烯酰胺为交联剂, 采用聚乙二醇甲基丙烯酸酯和 丙烯酰胺基苯嗍酸的共聚也能得到葡萄糖敏感的纳 米凝胶 ${ }^{[73]}$. 本课题组 ${ }^{[74]}$ 采用 “硫醇-烯”点击化学的一 步法, 以聚乙二醇单甲醚丙烯酸酯(mPEGA)、聚乙二 醇二丙烯酸酯(PEGDA)、四(3-颈基丙酸)季戊四醇酯 (QT)和AAPBA为原料制备了 PBA功能化的纳米凝胶. 采用茜素红(ARS)为苂光探针详细考察了葡萄糖分子 和ARS 竞争性与PBA特异性结合的性能, 证实纳米凝 胶中PBA基团的存在. 进一步以 ARS 为模型药物考察 了其葡萄糖敏感药物释放性能. 该纳米凝胶具有较好 的葡萄糖响应药物释放特性, 且药物释放不受多糖的 影响. 同时证实该纳米凝胶具有较好的生物相容性, 有望应用于药物自调式传递系统.

一种可注射的纳米凝胶(P(NIPAM-Dex-PBA))具有 较好的降血糖效果 ${ }^{[75]}$. 该纳米凝胶由PNIPAM和聚丙烯 酰胺基苯硼酸组成并采用葡聚糖-马来酸䣶(Dex-Ma) 交联, 具有较好的葡萄糖敏感胰岛素释放性能, 且纳米 凝胶中Dex含量越高, 葡萄糖敏感性能越好(图10(a)). 此纳米凝胶不仅具有较好的体外胰岛素释放特性, 糖 尿病大鼠体内降血糖效果更证实了其良好的葡萄糖 敏感性能. 如图 10(b)所示, 空白MG3纳米凝胶不具有 降血糖效果, 而载药 MG3 纳米凝胶具有较好的降血糖 效果, 且能够保持血糖稳定避免其波动, 其明显的降
血糖效果来源于纳米凝胶在糖尿病大鼠体内能够响 应血糖水平调控胰岛素的释放且所释放出的胰岛素 具有其原有的生物活性. 该可注射的纳米凝胶在胰岛 素自调式药物传递领域具有较好的应用前景.

聚氨基酸类材料用于葡萄糖敏感的纳米凝胶, 不 仅具有较好的生物相容性, 其可生物降解性能使其临 床应用具有更大的可能. 本课题组 ${ }^{[76]}$ 设计了己二酰氨 基苯硼酸交联的聚乙二醇单甲醚-block-聚( $\gamma$-苯甲基 $-L$-谷氨酸酯- $c o$-糖基化 $-L$-谷氨酸酯) (mPEG- $b$-P(BLG$c o-(\mathrm{PLG}-g-\mathrm{Glu}))$ ) 纳米凝胶(图11). 该纳米凝胶具有较 好的葡萄糖敏感胰岛素释放性能, 在 $2.0 \mathrm{mg} \mathrm{mL}^{-1}$ 葡萄 糖溶液中可快速且大量地释放出胰岛素, 而在无糖时 则缓慢释放胰岛素. 这主要是由于游离的葡萄糖分子 竞争性地与纳米凝胶中的PBA基团结合, 形成的苯硼 酸酯增加了聚氨基酸纳米凝胶的亲水性和空间体积, 从而促使胰岛素的快速释放. 生物相容性和生物可降 解的葡萄糖敏感聚氨基酸纳米凝胶在糖尿病的治疗 方面具有一定的临床应用价值.

同样采用竞争性结合, Kim等 ${ }^{[77]}$ 设计了一种新型 的聚氨基酸双层纳米凝胶. 该课题组通过开环聚合和 碳二亚胺耦合反应制备了海藻酸钠接枝的聚 ( $L$-谷氨 酸- $c o-N-3-L$ - 谷氨酰胺基苯硼酸(SA-PGGA)), 并将其 与改性的壳聚糖(GC)通过各向同性凝胶法和静电相 互作用制备了 GC/SA-PGGA双层纳米凝胶. 当葡萄糖 存在时, 由于葡萄糖小分子取代壳聚糖上的糖基元与 PBA结合, 使该纳米凝胶亲水性增加并释放出胰岛素 (图12(a)). 更重要的是, 该聚氨基酸纳米凝胶具有较好 的体内胰岛素可控释放性能. 通过框周注射葡萄糖使
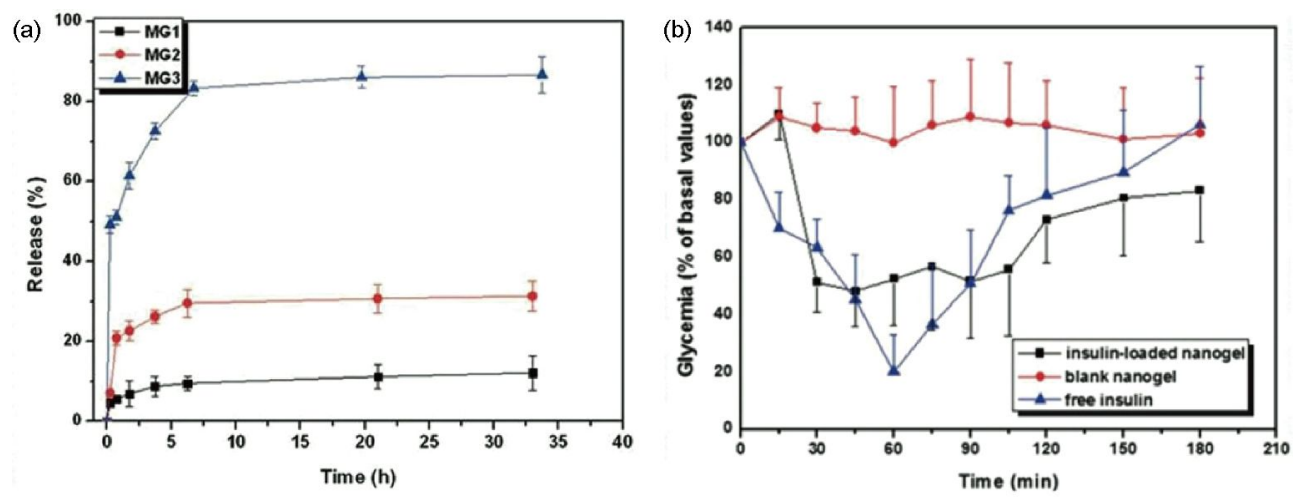

图 10 (a) MG1、MG2和 MG3 纳米凝胶在 $\mathrm{pH} 7.4 、 0.1 \mathrm{M}$ 的含 $2.0 \mathrm{mg} \mathrm{mL}^{-1}$ 葡萄糖的 $\mathrm{PBS}$ 中的胰岛素体外释放曲线(MG1、 MG2和MG3代表不同Dex含量的P(NIPAM-Dex-PBA)纳米凝胶, 且Dex的含量 $M G 1<M G 2<M G 3)$; (b) 皮下注射胰岛素(2.0 IU $\mathrm{kg}^{-1}$ )、载胰岛素 $\mathrm{MG} 3$ 纳米凝胶 (4.0 IU kg-1) 和空白MG3纳米凝胶后, 糖尿病大鼠的降血糖效果 ${ }^{[75]}$ (网络版彩图) 



图 11 PBA功能化的聚氨基酸纳米凝胶的葡萄糖敏感胰岛 素释放示意图 ${ }^{[76]}$ (网络版彩图)

小鼠血糖升高后, 将小鼠分组注射胰岛素和载胰岛素 纳米凝胶并尾静脉取血测试其血糖变化. 如图12(b)所 示, 除空白 GC/SA-PGGA纳米凝胶外, 在第二次注射 葡萄糖前所有实验组小鼠的血糖都逐步下降, 但载药 GC/SA-PGGA纳米凝胶处理的小鼠的血糖降低效果更 加明显. 第二次注射葡萄糖后, 载药GC/SA-PGGA纳 米凝胶处理的小鼠的血糖浓度也能够保持相对较低 的水平, 证实该纳米药物传递体系能够实现体内可逆 按需释放药物. 该GC/SA-PGGA双层纳米凝胶具有葡 萄糖响应的胰岛素自调式释放特性, 有望用于可控胇 岛素传输系统.

\subsection{PBA基杂化纳米粒子}

硅及其氧化物具有较好的生物相容性和表面可 修饰性, 被广泛应用于组织工程和药物传递领域 ${ }^{[78-80]}$. 特别是介孔二氧化硅(MSN)因其较大的孔隙率、可调
的介孔尺寸及可修饰性和生物相容性成为研究的焦 点 ${ }^{[81,82]}$. PBA功能化的MSN用于葡萄糖敏感药物载体 具有较好的药物可控释放特性 ${ }^{[8,84]}$.

$\mathrm{Li}$ 等 ${ }^{[85}$ 报道了一种蠕虫状的介孔二氧化硅用于 胰岛素的可控释放. 该MSN纳米粒子的聚合物刷由 AAPBA和NIPAM组成, 且采用Dex-Ma交联时 MSN纳 米粒子具有更持久的葡萄糖响应胰岛素释放性能. Liu 等 ${ }^{[86]}$ 采用MSN设计了新型的 $\mathrm{pH}$ 和葡萄糖双敏感的聚 合物交联网络包覆二氧化硅杂化纳米粒子. MSN 上 的聚合物刷 $\mathrm{P}(\mathrm{AA}-\mathrm{AGA})$ 是采用丙烯酸叔丁酯进行聚 合、水解以及糖基化得到的. 糖基化的 $\mathrm{P}(\mathrm{AA}-\mathrm{AGA})$ 聚合物刷经两端带PBA的小分子EPBA交联而包覆在 MSN的表面起到“盖子”的作用, 从而将模型药物Rd6G 包埋在MSN的空隙中. 当葡萄糖存在时, 由于游离的 葡萄糖小分子取代糖基化聚合物上的糖基元竞争性 与PBA结合而将聚合物“盖子”解交联, 从而将包埋在 MSN中的药物释放出来. 除葡萄糖敏感药物释放外, 该杂化纳米粒子的药物释放行为还受溶液 $\mathrm{pH}$ 的影响. 这主要是由于EPBA与聚合物刷上糖基元的特异性结 合在低于EPBA的 $\mathrm{p} K_{\mathrm{a}}$ 的酸的作用下受到破坏. 同一葡 萄糖浓度下, 溶液的 $\mathrm{pH}$ 越低, $\mathrm{Rd} 6 \mathrm{G}$ 的释放速率越快.

以外源性胰岛素作为模型药物研究葡萄糖敏感 药物载体释放性能的工作多见报道, 但针对促使胰腺 分泌内源性胰岛素的环腺苷酸(cAMP)的药物释放研 究较少. cAMP是胰腺 $\beta$ 细胞分泌胰岛素的重要调节因 子, 能够促使 $\mathrm{Ca}^{2+}$ 从线粒体释放到细胞中并进一步刺



图 12 (a) 不同葡萄糖浓度下的胰岛素累积释放曲线图; (b) 眼周注射空白GC/SA-PGGA纳米凝胶、胰岛素、载胰岛素GC/SA 纳米凝胶和载胰岛素 GC/SA-PGGA纳米凝胶后的小鼠血糖水平 ${ }^{[77]}$ (网络版彩图) 
激胰岛素的分泌. Lin课题组 ${ }^{[87]}$ 设计了MSN 新型纳米 粒子用于葡萄糖改性胰岛素(G-Ins)和cAMP的共担载. 如图13所示, G-Ins蛋白被固定在PBA功能化的MSN的 表面, 并作为“帽”将cAMP包埋在MSN的内部. 当有 葡萄糖存在时, 由于 G-Ins蛋白和MSN 上的PBA间的苯 硼酸酯络合物受到破坏, MSN的“帽”被打开, G-Ins和 cAMP响应葡萄糖刺激而被释放出来. 该纳米粒子还 具有较好的生物相容性、细胞摄取特性以及cAMP细 胞内释放特性, 有望用于生物分子的体内可控制释放.

除G-Ins蛋白可以作为MSN的“帽”, 能够响应葡萄 糖打开MSN的孔道而将药物释放出来外, 环糊精也可 以作为MSN的“开关”赋予其良好的葡萄糖敏感药物 释放性能. 将PBA修饰的MSN与功能化儿茶酚修饰的 $\beta$-环糊精 $(\mathrm{C}-\beta \mathrm{CD})$ 相结合, 可得到葡萄糖和 $\mathrm{pH}$ 双敏感 的纳米粒子 CD-PBA-MSN ${ }^{[88]}$. C- $\beta C D$ 上的 1,2 -二元醇 可以和 $\mathrm{PBA}$ 功能化的 MSN 上的PBA反应生成苯嗍酸 酯, 从而将MSN闭孔而将药物包埋在MSN的内部. 当 在葡萄糖溶液中或在酸性溶液中时, 苯嗍酸酯受到破 坏, C- $\beta C D$ 与 $M S N$ 发生脱离而将MSN的孔道打开从而 将药物释放出来. 该纳米药物传递的巧妙设计更易于 实现葡萄糖响应的药物“开-关”式释放特性.

Zhou等 ${ }^{[89}$ 制备了一种集葡萄糖检测、高载药量和 自调节药物释放于一体的多功能杂化纳米凝胶. 该杂化 纳米凝胶以 $\mathrm{Ag}$ 纳米粒子 $(10 \pm 3 \mathrm{~nm})$ 为核, 赋予其较强的 荧光性能, 以PBA功能化的聚(4-乙烯基苯硼酸-co-2-(二 甲基胺基)乙基丙烯酸酯) (P(VPBA-co-DMAEA))为壳 赋予其葡萄糖敏感性能(图14). 核-壳结构的杂化纳米 凝胶的性能受葡萄糖浓度的影响. 当葡萄糖浓度升高 时, PBA修饰的聚合物壳层膨胀, 不仅能将所担载的 胰岛素释放出来, 更重要的是聚合物壳层的膨胀致使 $\mathrm{Ag}$ 纳米粒子的理化环境发生变化, 从而使杂化纳米凝 胶的光信号依赖于葡萄糖浓度的变化而变化. 这种集 诊断和治疗于一体的多功能纳米粒子为胰岛素自调 式控制释放系统的设计提供了新的思路.

除 $\mathrm{Ag}$ 纳米粒子外, $\gamma-\mathrm{Fe}_{2} \mathrm{O}_{3}$ 也可以用于葡萄糖敏 感纳米药物传递系统. 新型Dex门控的多功能磁性 纳米粒子具有靶向细胞的葡萄糖响应药物释放性 能 ${ }^{[00]}$. 含有 $\gamma-\mathrm{Fe}_{2} \mathrm{O}_{3}$ 的磁性介孔二氧化硅经PBA修饰后 得到MMS-PBA纳米粒子, 并进一步经叶酸修饰得到 FA-MMS-PBA纳米粒子. MMS-PBA和FA-MMS-PBA 采用Dex将MMS上的“门”关闭而将药物封闭在纳米粒



图 13 葡萄糖敏感MSN传递系统用于G-Ins和cAMP的可控 制释放示意图 ${ }^{[87]}$ (网络版彩图)

子的内部, 继而得到 MMS-PBA-Dex 纳米粒子和 FA-MMS-PBA-Dex纳米粒子(图15(A)). 采用抗肿瘤药 物喜树碱考察了FA-MMS-Dex载药纳米粒子的葡萄 糖敏感性能, 并采用治疗II型糖尿病的甲苯磺丁艮为 模型药物, 考察了MMS-Dex载药纳米粒子的葡萄糖 敏感性能(图15(B)). 两种纳米粒子均具有较好的葡萄 糖敏感药物释放特性, 这主要是因为葡萄糖能够取代 Dex, 竞争性地与MMS上的PBA结合, 从而将“门”打 开将药物快速释放出来. 更重要的是, MMS-Dex载药 纳米粒子具有靶向肤腺 $\beta$ 细胞的葡萄糖敏感药物释放 性能, 而FA-MMS-Dex载药纳米粒子具有靶向癌细胞 的葡萄糖刺激药物释放性能. 该新型的Dex门控靶向 磁性纳米粒子为糖尿病/癌症的治疗提供了新的策略.

\section{5 其他纳米载体}

葡萄糖敏感纳米载体除了有高分子纳米胶束、 纳米囊泡、纳米凝胶和杂化纳米粒子外, 也有其他的 纳米载体形式. 准聚轮烷(PPRX) 也可以用于葡萄糖敏 感的药物载体. PPRX是一种通过非共价键相互作用 得到的超分子结构, 这种超分子结构是由不含封端剂 的链状大分子进入多个环糊精的疏水空腔组装而得 到的, 其中环糊精与大分子间的组装以其选择性、可 调控性和生物相容性等优势引起广泛关注 ${ }^{[1,92]}$.

Seki课题组 ${ }^{[93]}$ 通过PBA功能化的PEG (PBA-PEG) 和 $\gamma$-环糊精相互作用制备了葡萄糖敏感的 PPRX. 该 

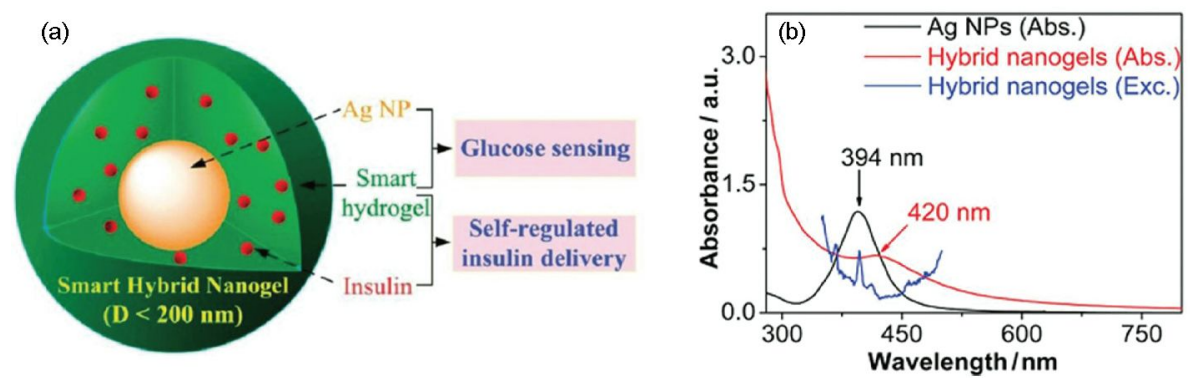

图 14 核-壳杂化纳米凝胶用于光学葡萄糖监测和自调式胰岛素的传递(a), 及其紫外-可见(UV-Vis)吸收光谱和激发光谱 (b) ${ }^{[89]}$ (网络版彩图)

(A)
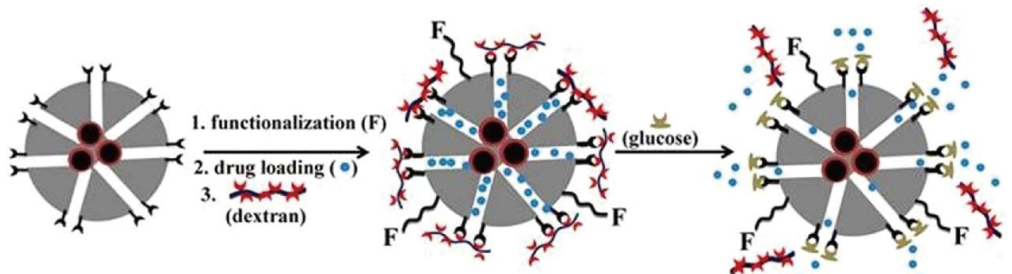

(MMS with gate)

(gate closed) (gate opened)
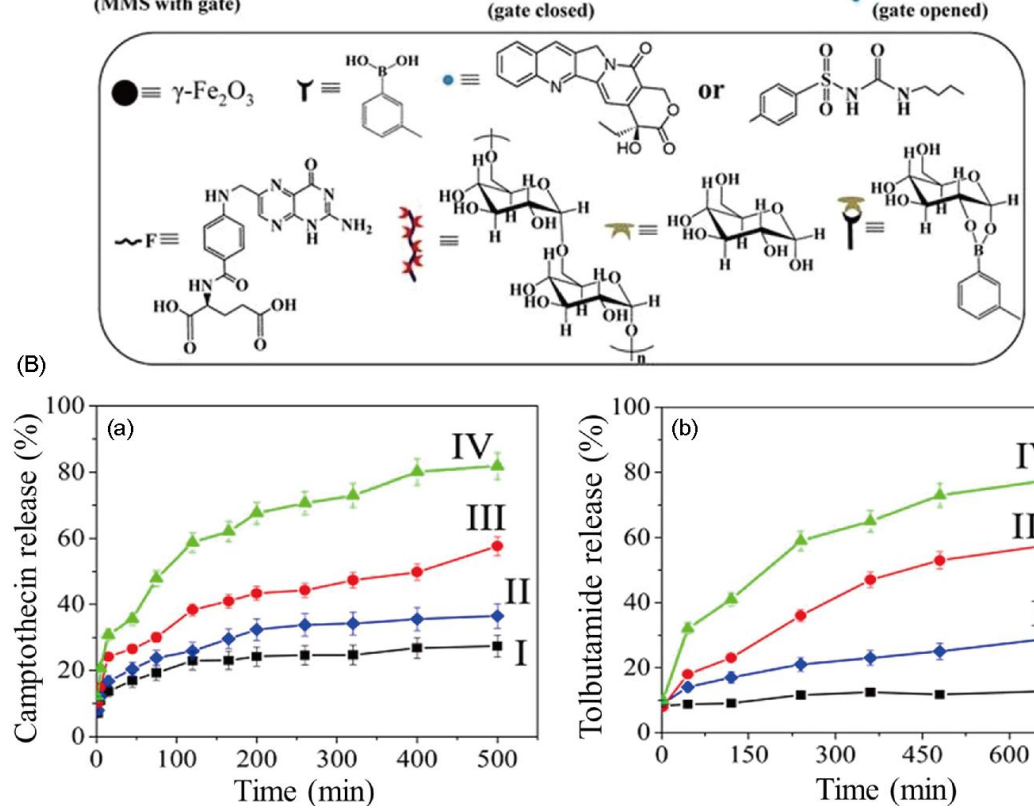

(B)

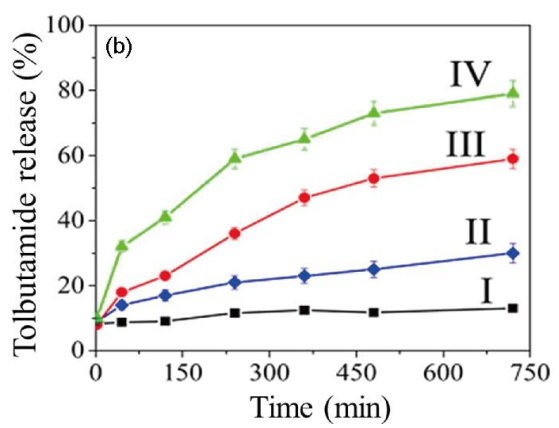

图 15 (A) Dex门控多功能介孔二氧化硅纳米粒子用于葡萄糖敏感药物传递; (B) pH 7.4时, 不同葡萄糖浓度下的FA-MMS-Dex 和MMS-Dex纳米粒子可控喜树碱(a)和甲苯磺丁脲(b)的释放(I, $0 \mathrm{mg} / \mathrm{mL} ; \mathrm{II}, 4 \mathrm{mg} / \mathrm{mL} ; \mathrm{III}, 10 \mathrm{mg} / \mathrm{mL} ; \mathrm{IV}, 50 \mathrm{mg} / \mathrm{mL})^{[90]}$ (网络版 彩图)

PPRX在 $D$-果糖或者 $D$-葡萄糖作用下能够解体. 虽然儿 茶酚也是带有 $1,2-$ 二元醇的小分子, 且儿茶酚与PBA具 有较高的亲和性, 但该PPRX在儿茶酚存在时不能解体, 对儿茶酚不具有敏感性能. 除此之外, 该课题组通过4羧基苯喼酸功能化的 $\gamma$-环糊精(PBA- $\gamma$-CyD)或3-羧基-
5-硝基苯硼酸功能化的 $\gamma$-环糊精 (NPBA- $\gamma$-CyD) 和菱修 饰的PEG (Naph-PEG) (图16)相互作用得到葡萄糖敏感 的 Naph-PEG/PBA- $\gamma$-CyD PPRX 和Naph-PEG/NPBA- $\gamma$ CyD PPRX ${ }^{[94]}$. Naph-PEG/PBA- $\gamma-C y D$ PPRX具有独特 的单链 PEG 穿过环糊精的结构, 且葡萄糖敏感基团 

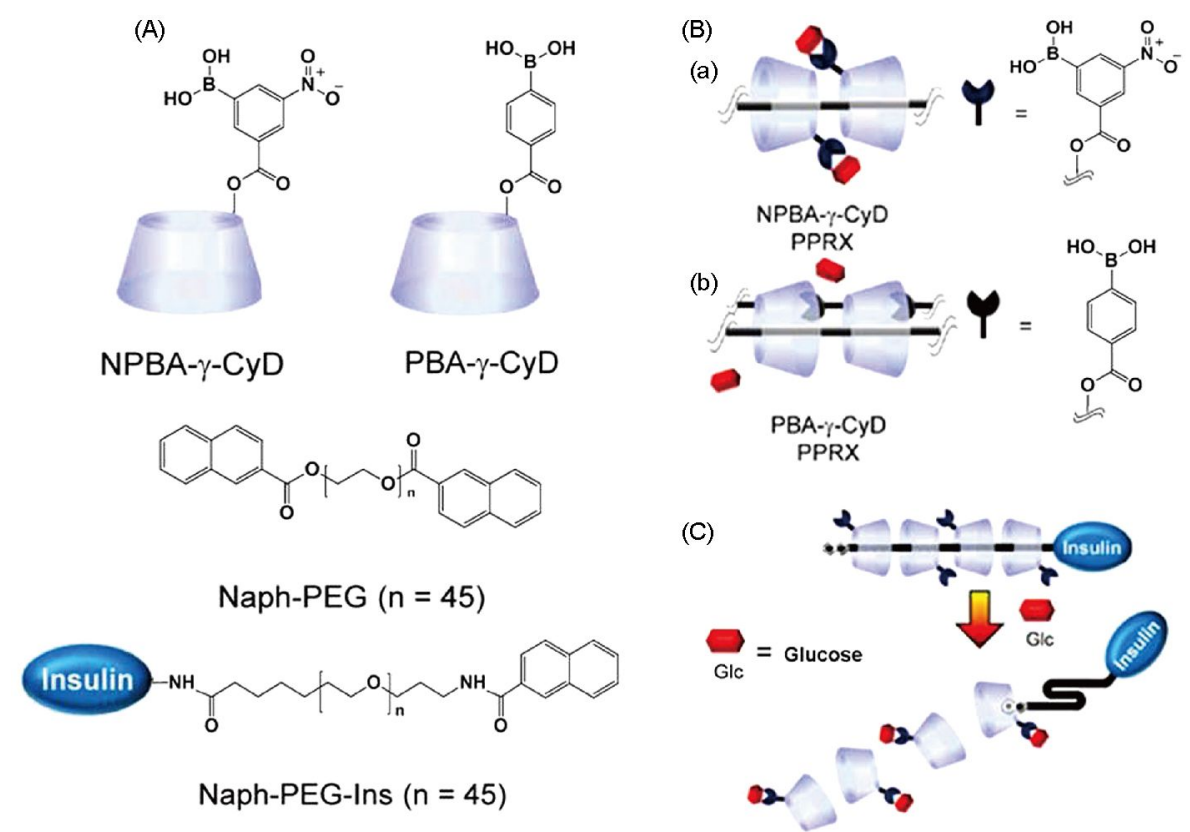

图 16 (A) NPBA- $\gamma-C y D 、 P B A-\gamma-C y D 、 N a p h-P E G$ 和Naph-PEG-Ins的化学结构; (B) (a) 葡萄糖敏感基团外漏的NPBA- $\gamma-C y D$ PPRX示意图; (b) 葡萄糖敏感基团在环糊精内腔的PBA- $\gamma$-CyD PPRX示意图; (C) Naph-PEG-Ins/NPBA- $\gamma$-CyD PPRX的葡萄糖 敏感胰岛素释放示意图 ${ }^{[94]}$ (网络版彩图)

PBA可以进入相邻环糊精的空腔. 进入相邻环糊精空 腔的PBA与葡萄糖分子间的特异性结合作用减弱, 降 低了PPRX的葡萄糖敏感性能(图16(B)-(a)). 相比较而 言, Naph-PEG/NPBA- $\gamma-C y D$ PPRX上的葡萄糖敏感基 团NPBA, 由于空间位阻较大不能进入到相邻环糊精的 空腔而裸露在外, 加之其与葡萄糖分子间的特异性结合 常数大, 该PPRX具有更好的葡萄糖敏感性能(图16(B)(b)). 末端修饰胰岛素分子的Naph-PEG (Naph-PEG-Ins) 结构如图 16(A) 所示, 将其与NPBA- $\gamma-C y D$ 相互作用得 到的Naph-PEG-Ins/NPBA- $\gamma$-CyD PPRX在葡萄糖作用 下解体而释放出 Naph-PEG-Ins (图 16(C)). 由于 PPRX 结构的不同, 与Naph-PEG-Ins/PBA- $\gamma$-CyD PPRX相比, 在同样条件下Naph-PEG-Ins/NPBA- $\gamma$-CyD PPRX则释 放出更多的Naph-PEG-Ins (图17), 开辟了 PPRX在葡萄 糖敏感药物传递领域的应用.

\section{3 结论与展望}

自调式控制葡萄糖敏感药物传递系统用于糖尿 病的治疗, 不仅可以减少注射次数, 更能够依据血糖 变化调控胰岛素的释放, 具有模拟人体胰腺分泌胰岛 素的生理模式, 极大地减少糖尿病患者的痛苦并提高
其生活质量. 基于PBA的葡萄糖敏感纳米药物载体更 成为本领域研究热点. 本文综述了 PBA基葡萄糖敏感 纳米药物传输体系的研究进展. 对PBA基葡萄糖敏感 纳米胶束、纳米囊泡、纳米凝胶以及杂化纳米粒子 的研究均取得了较大的进步, 但仍存在一些挑战限制 了葡萄糖敏感纳米粒子在糖尿病治疗中的临床应用. 这些挑战的克服将推动PBA基葡萄糖敏感纳米药物 传输体系的发展.

(1) 实现在生理 $\mathrm{pH}$ 具有可逆重复的葡萄糖敏感 药物释放性能仍是一个挑战. PBA及其衍生物的 $\mathrm{p} K_{\mathrm{a}}$ 远高于人体生理 $\mathrm{pH}$, 在 $\mathrm{pH}$ 7.4时不具有理想的葡萄糖 敏感性能. 尽管众多研究者为提高PBA在生理条件下 的葡萄糖响应能力采取了各种各样的措施, 如在PBA 上引入羧基、卤素、氨基或硝基基团以降低其 $\mathrm{p} K_{\mathrm{a}}$ 并 取得较好的研究结果, 但仍未得到能够真正实现在生 理条件下具有可重复且可逆的葡萄糖响应药物释放 的传输体系.

(2) 葡萄糖敏感药物控制释放体系给药的精准性 仍制约着药物传输体系的临床应用. 尽管高分子纳米 药物载体的体外释放实验证实其良好的葡萄糖敏感 性能, 但控制药物载体按需释放药物仍是一大难题. 

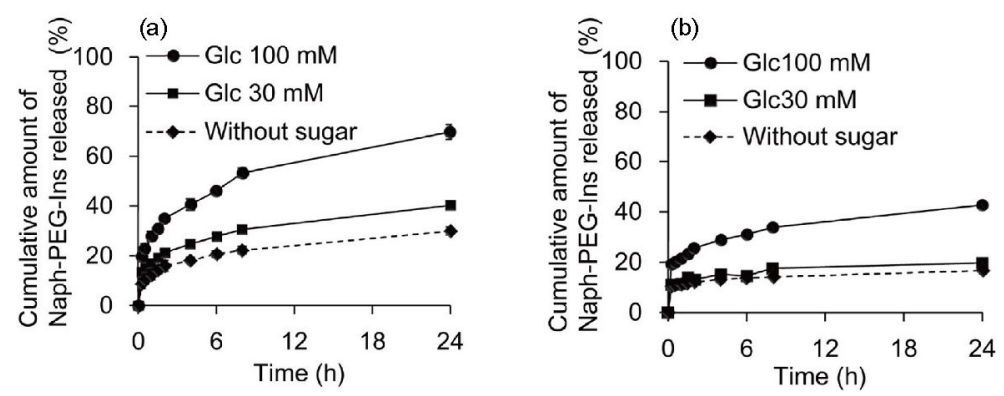

图 17 Naph-PEG/NPBA- $\gamma-C y D$ PPRX (a) 和Naph-PEG/PBA- $\gamma-C y D(b)$ 在不同葡萄糖浓度下的胰岛素释放性能 ${ }^{[94]}$ (网络版 彩图)

这种按需释放, 即在糖尿病患者血糖水平高时, 快速 释放一定量的胰岛素, 从而控制血糖浓度在正常水平, 但在正常人体血糖浓度时不释放药物从而保持血糖 水平稳定. 葡萄糖敏感药物载体真正实现人体胰腺 分泌胰岛素调控血糖的自调式药物控制释放任重而 道远.

(3) 保持所释放出的药物仍具有其生物活性也是 一个挑战. 在载药纳米载体制备过程中以及在药物释 放过程中, 不可避免地对生物活性药物分子造成影响, 从而影响有效给药剂量. 确保所释放出的药物具有其 初始药效十分必要.

(4) 确保药物载体具有较好的生物相容性和可生
物降解性, 在其真正用于糖尿病治疗时不具有毒副作 用并能及时排出体外也是一个研究重点. 糖尿病的治 疗是一个长期的过程, 用于治疗糖尿病的药物载体必 须安全、无毒, 且能够生物降解. 尽管用于葡萄糖敏 感药物自调式传递的药物载体具有较好的生物相容 性, 但聚丙烯酸类载体不具有生物降解性, 极大地限 制了其应用。

尽管仍面临着各种各样的挑战, 但葡萄糖敏感 PBA基高分子纳米粒子仍是糖尿病治疗药物可控释 放研究的焦点. 这种集实时调控血糖、高生物利用度 和病人顺从性好于一体的给药途径在大量研究者孜 孜不倦的努力下终将取得更大的进步.

\section{参考文献}

Kimura T, Takeuchi M, Nagasaki T, Shinkai S. Tetrahedron Lett, 1995, 36: 559-562

King H, Aubert RE, Herman WH. Diabetes Care, 1998, 21: 1414-1431

Uma MM, Sudarsanam D. Res J Biotechnol, 2012, 7: 72-79

Wild S, Roglic G, Green A, Sicree R, King H. Diabetes Care, 2004, 27: 1047-1053

Zhao Y, Ye W, Boye KS, Holcombe JH, Swindle R. Diabetic Med, 2009, 26: 61-69

Azagury A, Khoury L, Enden G, Kost J. Adv Drug Deliver Rev, 2014, 72: 127-143

Bakhru SH, Furtado S, Morello AP, Mathiowitz E. Adv Drug Deliver Rev, 2013, 65: 811-821

Soares S, Costa A, Sarmento B. Expert Opin Drug Deliver, 2012, 9: 1539-1558

Illum L. J Control Release, 2012, 161: 254-263

Wu Q, Wang L, Yu H, Wang J, Chen Z. Chem Rev, 2011, 111: 7855-7875

Guan Y, Zhang Y. Chem Soc Rev, 2013, 42: 8106-8121

Ma R, Shi L. Polym Chem, 2014, 5: 1503-1518

Matsumoto A, Ikeda S, Harada A, Kataoka K. Biomacromolecules, 2003, 4: 1410-1416

Wang J, Zhang Z, Wang X, Wu W, Jiang X. J Control Release, 2013, 168: 1-9

Wang X, Zhen X, Wang J, Zhang J, Wu W, Jiang X. Biomaterials, 2013, 34: 4667-4679

Zhao L, Xiao C, Wang L, Gai G, Ding J. Chem Commun, 2016, 52: 7633-7652

Li D, Chen Y, Liu Z. Chem Soc Rev, 2015, 44: 8097-8123

8 Farooqi ZH, Wu W, Zhou S, Siddiq M. Macromol Chem Phys, 2011, 212: 1510-1514 
Sato K, Yoshida K, Takahashi S, Anzai J. Adv Drug Deliver Rev, 2011, 63: 809-821

Hoare T, Pelton R. Macromolecules, 2007, 40: 670-678

Matsumoto A, Yamamoto K, Yoshida R, Kataoka K, Aoyagi T, Miyahara Y. Chem Commun, 2010, 46: 2203-2205

Matsumoto A, Ishii T, Nishida J, Matsumoto H, Kataoka K, Miyahara Y. Angew Chem Int Ed, 2012, 51: 2124-2128

Siegel RA, Gu Y, Lei M, Baldi A, Nuxoll EE, Ziaie B. J Control Release, 2010, 141: 303-313

Gao Y, Wong KY, Ahiabu A, Serpe MJ. J Mater Chem B, 2016, 4: 5144-5150

Dong Y, Wang W, Veiseh O, Appel EA, Xue K, Webber MJ, Tang BC, Yang XW, Weir GC, Langer R, Anderson DG. Langmuir, 2016, 32: 8743-8747

刘赣, 杨浩, 马如江, 史林启. 高分子学报, 2014, 9: 1161-1173

吕娟, 马如江, 史林启. 科学通报, 2016, 19: 2113-2123

Ding J, Chen L, Xiao C, Chen L, Zhuang X, Chen X. Chem Commun, 2014, 50: 11274-11290

Cambre JN, Roy D, Gondi SR, Sumerlin BS. J Am Chem Soc, 2007, 129: 10348-10349

Roy D, Cambre JN, Sumerlin BS. Chem Commun, 2008, 34: 2477-2479

Roy D, Sumerlin BS. ACS Macro Lett, 2012, 1: 529-532

Cambre JN, Roy D, Sumerlin BS. J Polym Sci A Polym Chem, 2012, 50: 3373-3382

Jiang G, Jiang T, Chen H, Li L, Liu Y, Zhou H, Feng Y, Zhou J. Colloid Polym Sci, 2015, 293: 209-215

Li L, Jiang G, Du X, Chen H, Liu Y, Huang Q, Kong X, Yao J. RSC Adv, 2015, 5: 75766-75772

Yao Y, Zhao L, Yang J, Yang J. Biomacromolecules, 2012, 13: 1837-1844

Yao Y, Wang X, Tan T, Yang J. Soft Matter, 2011, 7: 7948-7951

Wang B, Ma R, Liu G, Li Y, Liu X, An Y, Shi L. Langmuir, 2009, 25: 12522-12528

Ma R, Yang H, Li Z, Liu G, Sun X, Liu X, An Y, Shi L. Biomacromolecules, 2012, 13: 3409-3417

Yang H, Sun X, Liu G, Ma R, Li Z, An Y, Shi L. Soft Matter, 2013, 9: 8589-8599

Zheng D, An YY, Yang S, Wu W, Liu G, Yang C, Dan Y, Xu Z, Wu S. Int J Polym Mater Polym Biomater, 2013, 63: 115-122

Xu H, Yao Q, Cai C, Gou J, Zhang Y, Zhong H, Tang X. J Control Release, 2015, 199: 84-97

Matsumura Y. Adv Drug Deliver Rev, 2008, 60: 899-914

Chiang CH, Yeh MK. Curr Pharm Biotechnol, 2003, 4: 323-330

Zhao L, Ding J, Xiao C, Chen X, Gai G, Wang L. Acta Chim Sin, 2015, 73: 60-65

Zhao L, Ding J, Xiao C, He P, Tang Z, Pang X, Zhuang X, Chen X. J Mater Chem, 2012, 22: 12319-12328

Liu G, Ma R, Ren J, Li Z, Zhang H, Zhang Z, An Y, Shi L. Soft Matter, 2013, 9: 1636-1644

Guo Q, Wu Z, Zhang X, Sun L, Li C. Soft Matter, 2014, 10: 911-920

Guo H, Li H, Gao J, Zhao G, Ling L, Wang B, Guo Q, Gu Y, Li C. Polym Chem, 2016, 7: 3189-3199

Chai Z, Ma L, Wang Y, Ren X. J Biomater Sci Polymer Ed, 2016, 27: 599-610

Wang Y, Zhang X, Han Y, Cheng C, Li C. Carbohyd Polymers, 2012, 89: 124-131

Cheng C, Zhang X, Wang Y, Sun L, Li C. New J Chem, 2012, 36: 1413-1421

Guo Q, Zhang T, An J, Wu Z, Zhao Y, Dai X, Zhang X, Li C. Biomacromolecules, 2015, 16: 3345-3356

Jin X, Zhang X, Wu Z, Teng D, Zhang X, Wang Y, Wang Z, Li C. Biomacromolecules, 2009, 10: 1337-1345

Wu Z, Zhang S, Zhang X, Shu S, Chu T, Yu D. J Pharm Sci, 2011, 100: 2278-2286

Aguirre-Chagala YE, Santos JL, Aguilar-Castillo BA, Herrera-Alonso M. ACS Macro Lett, 2014, 3: 353-358

Wang Y, Chai Z, Ma L, Shi C, Shen T, Song J. RSC Adv, 2014, 4: 53877-53884

Wang Y, Chai Z, Wang N, Ren X, Gao M. J Biomater Sci Polymer Ed, 2015, 26: 617-628

Uchegbu IF. Expert Opin Drug Deliv, 2006, 3: 629-640

Zhang Y, Zhao W, Yang J, Hammouda B, Yang J, Cheng G. Eur Polymer J, 2016, 83: 173-180

Kim KT, Cornelissen JJLM, Nolte RJM, van Hest JCM. Adv Mater, 2009, 21: 2787-2791

Kim H, Kang YJ, Kang S, Kim KT. J Am Chem Soc, 2012, 134: 4030-4033

Yang H, Zhang C, Li C, Liu Y, An Y, Ma R, Shi L. Biomacromolecules, 2015, 16: 1372-1381

Yang H, Ma R, Yue J, Li C, Liu Y, An Y, Shi L. Polym Chem, 2015, 6: 3837-3846

Shi D, Ran M, Huang H, Zhang L, Li X, Chen M, Akashi M. Polym Chem, 2016, 7: 6779-6788

Wu Q, Wang L, Yu H, Chen Z. Polym Sci Ser A, 2012, 54: 209-213

Ancla C, Lapeyre V, Gosse I, Catargi B, Ravaine V. Langmuir, 2011, 27: 12693-12701

Farooqi ZH, Khan A, Siddiq M. Polym Int, 2011, 60: 1481-1486 
Wang D, Liu T, Yin J, Liu S. Macromolecules, 2011, 44: 2282-2290

Hazot P, Delair T, Elaïssari A, Chapel JP, Pichot C. Colloid Polym Sci, 2002, 280: 637-646

Xing S, Guan Y, Zhang Y. Macromolecules, 2011, 44: 4479-4486

Liu P, Luo Q, Guan Y, Zhang Y. Polymer, 2010, 51: 2668-2675

Wang C, Xing Z, Yan J, Li L, Zhao H, Zha L. Chin J Mater Res, 2012, 26: 44-48

Li L, Jiang G, Jiang T, Huang Q, Chen H, Liu Y. J Polym Mater, 2015, 32: 77-84

Zhao L, Xiao C, Ding J, He P, Tang Z, Pang X, Zhuang X, Chen X. Acta Biomater, 2013, 9: 6535-6543

Wu Z, Zhang X, Guo H, Li C, Yu D. J Mater Chem, 2012, 22: 22788-22796

Zhao L, Xiao C, Ding J, Zhuang X, Gai G, Wang L, Chen X. Polym Chem, 2015, 6: 3807-3815

Lee DY, Choe K, Jeong YJ, Yoo J, Lee SM, Park JH, Kim P, Kim YC. RSC Adv, 2015, 5: 14482-14491

McInnes SJP, Voelcker NH. Future Med Chem, 2009, 1: 1051-1074

Li J, Qin X, Yang Z, Qi H, Xu Q, Diao G. Talanta, 2013, 104: 116-121

Wang X, Tan L, Yang Y. Acta Chim Sin, 2016, 74: 303-311

Zou Z, He D, Cai L, He X, Wang K, Yang X, Li L, Li S, Su X. ACS Appl Mater Interf, 2016, 8: 8358-8366

Trewyn BG, Giri S, Slowing II, Lin VSY. Chem Commun, 2007, 114: 3236-3245

Lapeyre V, Renaudie N, Dechezelles JF, Saadaoui H, Ravaine S, Ravaine V. Langmuir, 2009, 25: 4659-4667

Zhang L, Xu Y, Yao H, Xie L, Yao J, Lu H, Yang P. Chem Eur J, 2009, 15: 10158-10166

Sun L, Zhang X, Zheng C, Wu Z, Li C. J Phys Chem B, 2013, 117: 3852-3860

Tan L, Yang MY, Wu HX, Tang ZW, Xiao JY, Liu CJ, Zhuo RX. ACS Appl Mater Interf, 2015, 7: 6310-6316

Zhao Y, Trewyn BG, Slowing II, Lin VSY. J Am Chem Soc, 2009, 131: 8398-8400

Yilmaz MD, Xue M, Ambrogio MW, Buyukcakir O, Wu Y, Frasconi M, Chen X, Nassar MS, Stoddart JF, Zink JI. Nanoscale, 2015, 7: 1067-1072

Wu W, Mitra N, Yan ECY, Zhou S. ACS Nano, 2010, 4: 4831-4839

Sinha A, Chakraborty A, Jana NR. ACS Appl Mater Interf, 2014, 6: 22183-22191

Liao X, Chen G, Liu X, Chen W, Chen F, Jiang M. Angew Chem Int Ed, 2010, 49: 4409-4413

Wang Y, Wang H, Chen Y, Liu X, Jin Q, Ji J. Chem Commun, 2013, 49: 7123-7125

Seki T, Namiki M, Egawa Y, Miki R, Juni K, Seki T. Materials, 2015, 8: 1341-1349

Seki T, Abe K, Egawa Y, Miki R, Juni K, Seki T. Mol Pharm, 2016, 13: 3807-3815 


\title{
Phenylboronic acid-functionalized polymer nanocarriers for glucose-triggered drug release
}

\author{
Li Zhao $^{1,3 \dagger}$, Qiongwei Huang ${ }^{2 \dagger}$, Liyan Wang $^{1}$, Guangqing Gai ${ }^{1 *}$, Shanshan Xiao ${ }^{1}, \mathrm{Fei}^{1}{ }^{1}$, Jianxun \\ Ding $^{3 *}$ \\ ${ }^{1}$ College of Material Science and Engineering, Jilin Jianzhu University, Changchun 130118, China \\ ${ }^{2}$ Department of Endocrinology, China-Japan Union Hospital of Jilin University, Changchun 130033, China \\ ${ }^{3}$ Key Laboratory of Polymer Ecomaterials, Changchun Institute of Applied Chemistry, Chinese Academy of Sciences, Changchun 130022, China \\ $\dagger$ These authors contributed equally to this work \\ *Corresponding authors (email: gaigq@163.com; jxding@ciac.ac.cn)
}

\begin{abstract}
Recently, glucose-sensitive polymer drug delivery systems have attracted growing attention, which control drug release automatically and continuously depending on the change of glucose concentration. Glucose-sensitive drug nanocarriers based on phenylboronic acid (PBA) have shown promising potential applications in the therapy of diabetes due to the great stability, long term storability, and reversible glucose-sensitivity. Particularly, the PBA-based glucose-sensitive polymer nanocarriers have become the research focus in this field. This review covers the recent development in the PBA-based glucose-sensitive nanocarriers, such as namomicelles, nanovesicles, nanogels, and hybrid nanoparticles. The development trend and application prospect of PBA-functionalized polymer nanocarriers in self-regulated drug delivery are also predicted.
\end{abstract}

Keywords: glucose-sensitivity, phenylboronic acid, polymer, nanocarrier, drug delivery, diabetes therapy

doi: $10.1360 / \mathrm{N} 032017-00021$ 